Article

\title{
The "Zero Point" and the Autonomous Nervous System. Reverse analysis, syllogism and genetic. Possible correlations with the Covid19 virus. A new perspective.
}

\author{
Carlo Brogna \\ Achieved both titles in Medicine (Salerno University Medical school) and Dental School (Pescara University \\ Dental School); c.brogna@libero.it \\ Specialist in oral facial pain (Pescara University Dental School) and expert in oral surgery (few period at \\ NJDS-Usa); \\ Attended in Neurosurgery (thesis in "The trochlear nerve: microanatomic and endoscopic study" - Salerno \\ University Medical school).
}

\begin{abstract}
Acetylcholine (ACh) is one of the best characterized neurotransmitters. Its central roles in the cholinergic areas and central nervous system (CNS) and peripheral (PNS) synapses are well known. It was the first molecule identified as a neurotransmitter and appears to be phylogenetically one of the oldest signaling molecules. In fact, ACh was detected in bacteria, protozoa, fungi, algae and primitive plants, indicating that the cholinergic system was widely distributed in living organisms before its appearance in the nervous system. The autonomous nervous system (ANS) is an integral part animal kingdom history. It determines fight-escape reactions as well as outlining the vital and cognitive functions bio-organization rhythms. The virus (Covid-19) extraneous effects acting on it could help to better understand its functions. Through a new deductive and inductive the path axioms are developed and an absurd hypothesis, "natural genetic recombination between different species" is put forward, trying to verify a correlation, tracing what could be the genesis, the "zero-point", crucial and fundamental moment. A positive correlation with different toxins is obtained and the double pathogenic mechanism of Covid-19 theory is proposed. The process allows us to outline the possibility about toxic-like factors presence.
\end{abstract}

Keywords: Covid-19; Blastn; toxins protein; ACh; aChE; BuChE; CBP; Zero Point; bungaro-toxins; phospholipase A2; similar prothrombin activator protein

\section{Introduction.}

Beta-coronaviruses are capsuled and have a single RNA filament with a positive sense, ranging in size from 26 to 32 kilobas. The genome encodes for structural, non-structural and ancillary proteins. In 2002, severe acute coronavirus syndrome (SARS-CoV) emerged in China. The SARS-CoV outbreak lasted 8 months and led to 8,098 confirmed human cases worldwide,774 (9.5\%) Fatal. About 10 years later, another highly pathogenic human coronavirus, Middle East Respiratory Syndrome (MERS$\mathrm{CoV})$, appeared in the Kingdom of Saudi Arabia affecting 2,260 cases in 27 countries and 803 were fatal. Many human and animal coronaviruses appear to originate from a bat species variety. Sequencing with new-generation techniques, there are about 200 new coronaviruses. SarS-CoV studies suggested Himalayan palm civets and raccoon as the most likely hosts responsible for human transmission. The bats role has been hypothesized later. Dromedary camels are a natural host and tank for MERS-CoV, but primary MERS-CoV cases have had no contact history with camels or infected individuals. HCoV-NL63 was first identified in 2004 in a pediatric patient with bronchiolitis 
and since then it has been learned that the virus causes about 1-9\% common colds each year and has been circulating in humans for centuries. Although viruses like to HCoV-NL63 have been identified in bats, they have fairly distant sequences, suggesting a possible intermediate host. HCoV-229E, which causes the common cold, appears to have its origins even in bat species. HCoV-229E-related viruses have been found in hipposider bats in Kenya and Ghana. In 2007, an alpha-coronavirus was identified in a respiratory disease epidemic in the United States alpacas. Genomic changes Evidence in the HCoV$229 E$ evolution, between bat and alpacas and subsequently between alpacas and human evolution has been identified. Bats remain the main evolutionary reservoirs and ecological drivers of CoV diversity (1). SARS genomic sequences are related to CoVid-19 and other animal species. The epidemic virus initial stages in Wuhan, December 2019, are probably related to the fish market (2). Some studies suggest the bat as a potential host (3). The SARS-CoV-2 genomic sequence is $96.2 \%$ identical to the CoVRaTG13 sequence, as well as having a 79.5\% genomic identity with SARS-CoV. The bat is suspected as a natural host and SARSCoV-2 may have been transmitted to humans by an unknown intermediate host. It is found that SARS-CoV-2 uses the angiotensin 2 conversion enzyme receptor as well as SARS$\mathrm{CoV}$ and establishes an avid receptor link with pneumocytes cells $(4,12)$. Ace2 pneumocyte regulates both cross-species and transmission between humans (11). The coronavirus surface S-glycoprotein virion of the binds to the ACE2 receptor $(13,14,15,16$,$) . Ace2-receptor adherence efficiency is 10$ to 20 times higher than SARS-CoV $(14,15,16)$. The protein sequences alignment and phylogenetic analysis, considering that similar residues of the ACE receptor have been observed in many species, suggest more alternative intermediate hosts possibilities, such as turtles, pangolin and snakes. The interhuman transmission of SARS-CoV-2 takes place mainly among family members, including relatives and friends $(5,6)$. The Wuhan-Hu-1 Coronavirus (WHCV) complete genome, a SARSCoV-2 strain, is $29.9 \mathrm{~kb}(6)$ while SARS-CoV and MERS-CoV has $27.9 \mathrm{~kb}$ and $30.1 \mathrm{~kb}$ positive RNA genomes, respectively (7). An open reading frames (ORF) variable number (six to eleven) the SARSCoV-2 genome contains. The $2 / 3$ RNA viral genome, the first ORF (ORF1a/b), translates two polyproteins, pp1a and pp1ab, and encodes 16 non-structural proteins (NSPs) while the remaining ORFs encode ancillary and structural proteins. The remaining part encodes essential structural proteins (glycoprotein spike (S), small envelope protein (E), matrix protein (M) and nucleocapsid protein (N)) and several ancillary proteins who interfere with the human immune response $(8,9)$. There are genomic and phylogenetic similarities with SARS-CoV in the S-glycoprotein gene and receptorbinding (RBD) domain, which indicates human direct transmission capacity. Mutations in NSP2 and NSP3 plays a role in the infectious capacity and differentiation mechanism of SARS-CoV-2 (9). The viral RNA receptor gene inducible retinoic acid I (RIG-I), a differentiated melanoma cytosolic receptor associated with gene 5 (MDA5) and the cyclic nucleotide-transferase synthesizer GMP-AMP (cGAS) are responsible for the recognition of RNA and DNA in cytoplasm. These complexes lead to the nuclear factor-B transcription (NF-B) and the interferon factor 3 (IRF3) activation, and regulators the interferons type I (IFN-z) and pro-inflammatory cytokines, including IL-1, IL-2, IL-4, IL-7, IL-10, IL-12, IL-13, IL-17, GCSF, Macrophage Colony Stimulation Factor (MCSF), IP-10, MCP-1, MIP-1, Hepatocyte Growth Factor (HGF), IFN-z and TNF-z, production (17-18-19-20).

In Wuhan, the $73 \%$ patients infected (average age 49 years) were men, 32\% had underlying conditions including diabetes $(20 \%)$, hypertension $(15 \%)$ cardiovascular disease $(15 \%)$. The most common symptoms onset were: fever $(98 \%)$, cough $(76 \%)$ and myalgia or fatigue (44\%). Less common symptoms: sputum $(28 \%)$, headaches $(8 \%)$, hemoptysis $(5 \%)$ diarrhea $(3 \%)$. The average time from the first symptom to breathlessness was 5 days, to the hospitalization 7 days and to ARDS of 8 days. Lymphopenia was present in the $63 \%$. All patients had interstitial pneumonia with abnormal chest CT results. Complications included: acute respiratory distress syndrome (29\%), RNA grow (15\%), acute heart damage $(12 \%)$, secondary infection $(10 \%)$.Most patients received antiviral therapy (oseltamivir, 89.9\%) and many received antibacterial therapy (moxifloxacin, 64.4\%; ceftriaxone, $24.6 \%$; azithromycin, $18.1 \%$ and glucocorticoid therapy, $44.9 \%$ ). Between $26.1 \%$ and $32 \%$ patients needed intensive care due to complications, including acute respiratory distress syndrome (61.1\%), arrhythmia (44.4\%) shock (30.6\%). The latter had higher plasma levels of IL2, IL7, IL10, GSCF, IP10, MCP1, MIP1A, and TNF. Patients treated in intensive care, compared to patients not treated in 
intensive care, were older (median age, 66 years vs. 51 years), and $72.2 \%$ had underlying comorbidity (23.24).

The Guidelines ( $\mathrm{VI}^{\circ}$ edition) recommended antivirals, including IFN-z, lopinavir/ritonavir, ribavirin, chloroquine phosphate (30). As in the past, infusion of convalescent blood products (CBP) has been practiced in Wuhan. They can derive from whole blood or plasma (convalescent whole blood, convalescent plasma or convalescent serum, healed pooled human immunoglobulin (Ig) a and immunized patient, high-title human Ig, polyclonal or monoclonal antibodies), like Ebola, Sars, Mers protocols (22).

Wei Ji et al. 2020 suggest that 2019-nCoV has a very similar bat coronaviruses genetic information and that there is a more similar sequence to snake bias; two snakes' types - the Bungarus multicinctus (multi-band krait) and the Naja atra (Chinese cobra).

\section{Materials and Methods.}

Collected and analyzed the data, published from January 2020 to 28.03.2020, 10 safe and certain axioms are postulated (Table 1) and the absurd hypothesis: "The covid-19 is not just a virus is formulated. This analysis is called as "Reductio Ad Absurdum".

\section{Axioms (Table 1):.}

\begin{tabular}{ll}
\hline 1. & No study has denied an intermediate host. \\
\hline 2. & The Covid-19 virus is respiratory. \\
\hline 3. & The Covid-19 virus has an interhuman spread. \\
\hline 4. & There isn't the same out-come in covid-19 patients about age \\
\hline 5. & There isn't the same out-come in covid-19 patients about previous health status. \\
\hline 6. & There isn't the same out-come in covid-19 patients about gender. \\
\hline 7. & There is contracting Covid-19 greater individuals' group risk. (Health workers- patients with \\
comorbidity- elderly and others for unknown reasons). \\
\hline 8. & the covid-19 cans be also contracted by direct contact. \\
\hline 9. & No study verified other unknown covid-19 properties and/or proteins acquired by the intermediate \\
host. & No study has denied the traits and/or characteristics and/or proteins (or RNA genomic sequences, \\
\hline 10. & Micro Rna, enzymes or molecules) acquisition possibility by the intermediate host. \\
\hline
\end{tabular}

The Questions to Be Met.

1. Could the Covid-19 virus have acquired, in its genomic set, sequences that transcribe factors (micro-Rna? Enzymes? Proteins? Toxins? else?) like those in the intermediate host? A: Unknown? Cross species genomic recombination? (21)

2.Is there another laboratory data besides the useful nasal or oral-pharyngeal swab at any way? A: Unknown

3. If the absurd hypothesis were true, would the treatments and therapies be enough?

The analysis.

A genomic sequence blast-alignments series were performed to verify the analyzed data match and the "genesis", the "zero point". Alignments were made through the BLAST-algorithm (blastn ncbi.nlm.nih.gov) and were practiced between the Covid19 (id. NC_045512.2.), the pangolin ("Manis pentadactyla") and the snakes of the Bungarus multicinctus, fasciatus, the Naja Atra. The snakes produce toxins and are suggested by Wej Ji as intermediate host. The acid secreted by the pangolin's glands (KN005617.1, blast-n Figure 5) had no use in medicine while snake toxins were particularly studied, sequenced, recombined, reproduced and used for analysis of cholinergic receptor mechanisms, as precursors to many drugs including Captopril and congeners, such as antithrombotic drugs. The toxins originated from early positive results for a significant correlation between these molecules and the Covid-19 genome, is defined by the match with a low e-value and a high identity rate. 


\begin{tabular}{|c|c|c|c|c|c|c|c|c|}
\hline \multicolumn{2}{|c|}{ descrixioni } & ritionat & & & & & & \\
\hline \multicolumn{3}{|r|}{ Sequenze che producono attineamenti significativi } & searica $\sim$ & \multicolumn{3}{|c|}{ Gestisci colonne - } & Mostr & $100 \checkmark$ \\
\hline & \multicolumn{3}{|c|}{ D seleziona tutto as sequense selesionose } & \multicolumn{2}{|c|}{ Gentank } & \multicolumn{3}{|c|}{ Albero dei rnuteati della diskanzo } \\
\hline & & Descrielone & manosine & Printerpos & Conery & $\begin{array}{l}\text { Valore } \\
\text { E. }\end{array}$ & Peet & ecteswiones \\
\hline & $\approx$ & 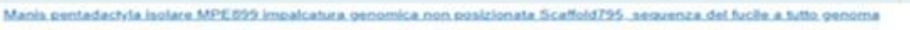 & soo & soo & as & Q."1 & 06365 & 000050127 \\
\hline & ص & 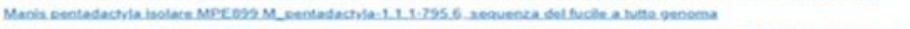 & soo & soo & os & 0.11 & $0636 \%$ & 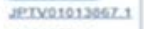 \\
\hline & $\approx$ & 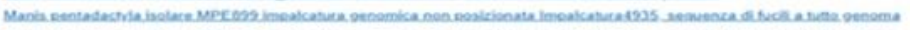 & 473 & 473 & os & 14 & a7sos & covetessus \\
\hline & $\Xi$ & 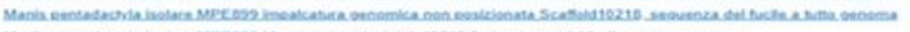 & 473 & 473 & os & 14 & eess & knows?10.1. \\
\hline & $\Xi$ & 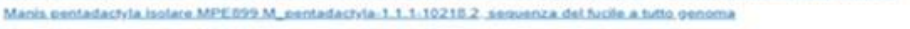 & 473 & 473 & os & 14 & $06.6 \%$ & aptveroesoss. 1 \\
\hline & $\Xi$ & 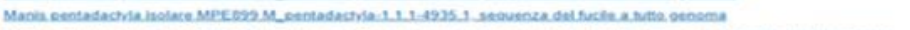 & 43 & A73 & os & 14 & orsos & PTVeresasss.1. \\
\hline & 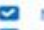 & 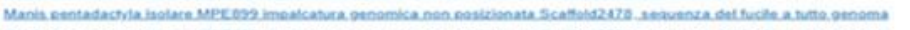 & 46.4 & 464 & os & 1.4 & $9333 \times$ & 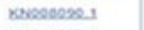 \\
\hline & $\Xi$ & 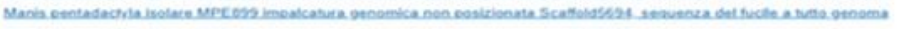 & 264 & 464 & os & 14 & 76925 & 801011202.1 \\
\hline & $\Xi$ & 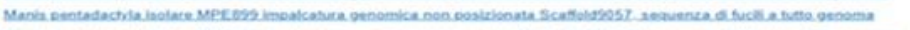 & 26.4 & 464 & os & 14 & 00.195 & veversing. \\
\hline & 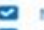 & 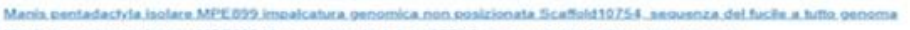 & 46.4 & 46.4 & os & 14 & 93305 & Kave16230.1 \\
\hline & ص & 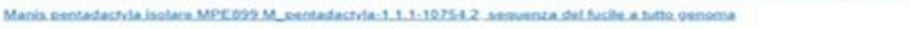 & 46.4 & 46.4 & os & 14 & 93305 & 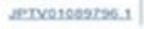 \\
\hline \multicolumn{3}{|r|}{ 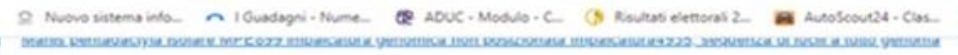 } & \multicolumn{3}{|c|}{ \& Molvices-Fiee M } & novo & $\begin{array}{l}\text { Numolipos. } \\
\text { uriwa }\end{array}$ & BS. OR \\
\hline 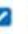 & \multicolumn{2}{|r|}{ 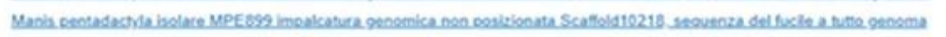 } & 473 & 473 & ors & 1.4 & $2696 \mathrm{~S}$ & KN215716.1 \\
\hline 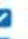 & \multicolumn{2}{|r|}{ 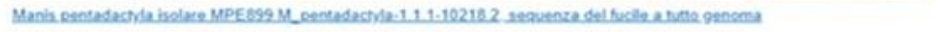 } & 473 & 473 & or & 1.4 & 86965 & sprvereosess.1 \\
\hline 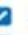 & \multicolumn{2}{|r|}{ 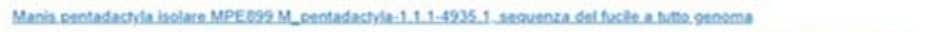 } & 473 & 473 & or & 1.4 & 87.505 & jPTVeres3sss.1 \\
\hline 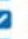 & \multicolumn{2}{|r|}{ 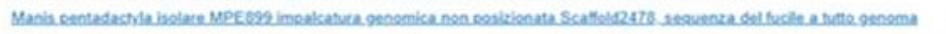 } & 46.4 & 46.4 & os & 1.4 & $9333 \%$ & neveoesese.1 \\
\hline 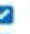 & \multicolumn{2}{|r|}{ 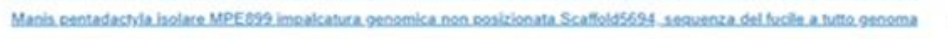 } & 464 & 46.4 & or & 1.4 & $76.92 \mathrm{~s}$ & kNov112at.1. \\
\hline 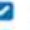 & \multicolumn{2}{|r|}{ 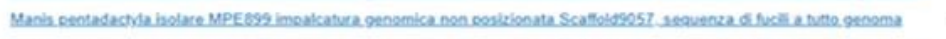 } & 46.4 & 46.4 & os & 1.4 & $89.19 \mathrm{~s}$ & KNo145TH.1 \\
\hline 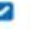 & \multicolumn{2}{|r|}{ 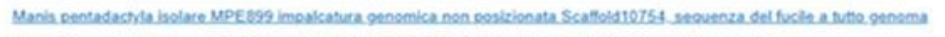 } & 46.4 & 464 & os & 1.4 & $93.33 \mathrm{~s}$ & BNo15238.1 \\
\hline 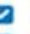 & \multicolumn{2}{|r|}{ 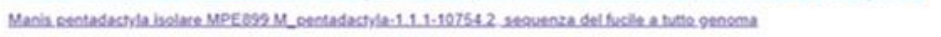 } & 46.4 & 46.4 & or & 1.4 & 93335 & aptveresgrgs.1 \\
\hline 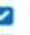 & \multicolumn{2}{|r|}{ 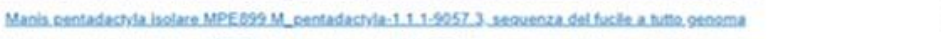 } & 464 & 46.4 & or & 14 & $89.19 \%$ & aprvereecren \\
\hline 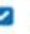 & \multicolumn{2}{|r|}{ 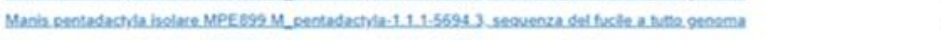 } & 46.4 & 46.4 & ors & 14 & 76925 & aptveres20061 \\
\hline 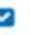 & \multicolumn{2}{|r|}{ 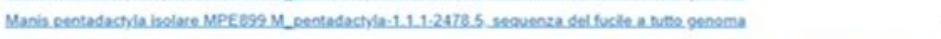 } & 46.4 & 46.4 & os & 1.4 & 93335 & setvore.23652.1 \\
\hline 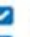 & & 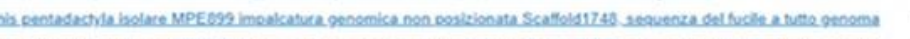 & 45.5 & 45.5 & os & 47 & $96.30 \mathrm{~s}$ & KNeens3:1 \\
\hline 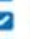 & & 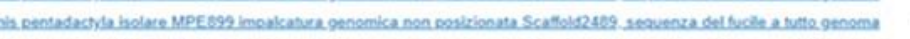 & 455 & 45.5 & os & 47 & 96.305 & seveosiens \\
\hline 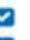 & & 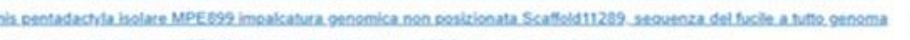 & 45.5 & 455 & os & 47 & 90.625 & kNe167491 \\
\hline 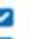 & & 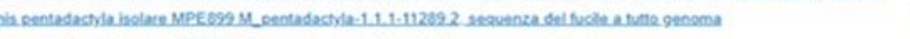 & 455 & 45.5 & or & 47 & $90.62 \mathrm{~s}$ & aptveregzes:1 \\
\hline 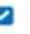 & & 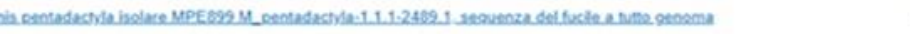 & 455 & 45.5 & os & 47 & $96.30 \mathrm{~s}$ & aptVo1032797.1 \\
\hline 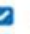 & & 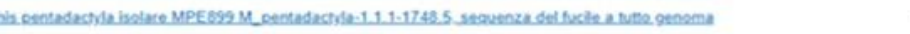 & 45.5 & 45.5 & or & 47 & $96.30 \mathrm{~s}$ & PPTVere2523s.1 \\
\hline 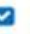 & & 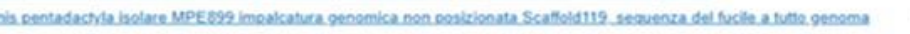 & 446 & 446 & $0 \%$ & 47 & $85.11 \%$ & kNoes735.1 \\
\hline 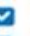 & & 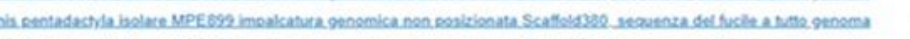 & 446 & 446 & os & 47 & 93.105 & kNovesg97.1 \\
\hline 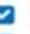 & & 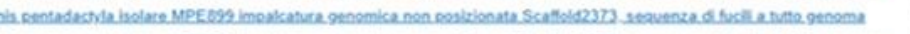 & 446 & 446 & or & 47 & 73975 & KNoeT2ar.1. \\
\hline 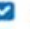 & & 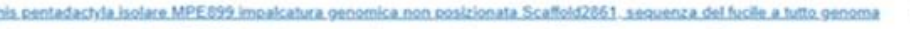 & 446 & 446 & os & 47 & $8462 \%$ & 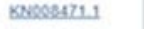 \\
\hline 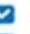 & & 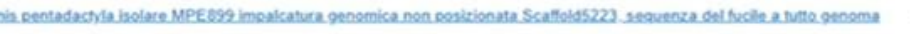 & 446 & 446 & os & 47 & $93.10 \mathrm{~s}$ & KNo10818.1 \\
\hline 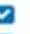 & & 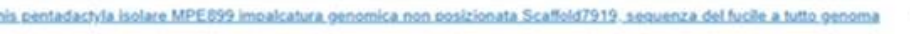 & 446 & 446 & os & 47 & 93.105 & Kave13451.1 \\
\hline 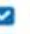 & & 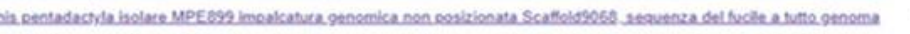 & 446 & 446 & os & 47 & 100.005 & kneresses.1. \\
\hline 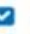 & & 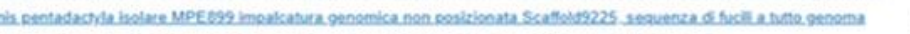 & 446 & 446 & os & 47 & $100.00 \%$ & KNe147421 \\
\hline 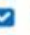 & & 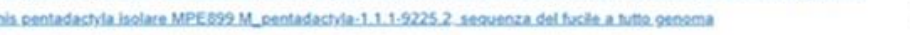 & 446 & 446 & os & 47 & $100.00 \%$ & aptrotesizes: \\
\hline 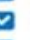 & & 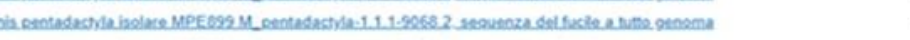 & 446 & 446 & os & 47 & $100.00 \%$ & aptvereedezse.1 \\
\hline 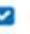 & & 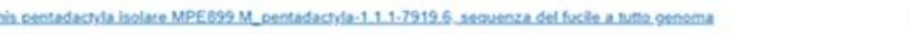 & 446 & 446 & or & 47 & $93.10 \mathrm{~s}$ & sprverezast3.1 \\
\hline 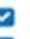 & & 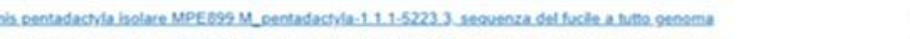 & 446 & 446 & os & 4.7 & $93.10 \mathrm{~s}$ & ietveressseg.1 \\
\hline 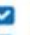 & & 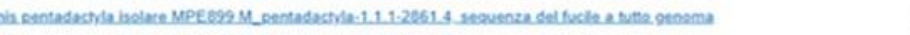 & 446 & 446 & os & 47 & 84625 & Prveress205.1 \\
\hline 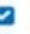 & & 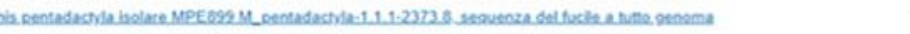 & 446 & 446 & os & 47 & $7397 \%$ & aptrve1e31651.1 \\
\hline 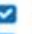 & & 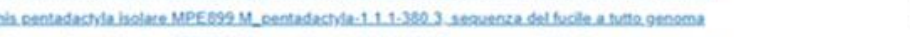 & 446 & 446 & $0 \%$ & 47 & 93.105 & aptvoreqzases \\
\hline$\square$ & & 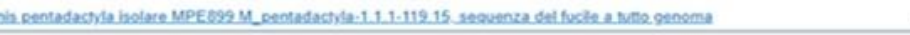 & 446 & 446 & os & 47 & 85.11s & sprvereazes21 \\
\hline
\end{tabular}

Figure 5. ("Manis pentadactyla").

The best toxins' correlations are: B. multicinctus peptide 1 similar to neurotoxin (NL1) (X64593.1), Query cover 32\%, e-value 0.5, 100\% identity percentage (Figure 1A);

Cardiotoxin VII Naja atra (id. U42584.1), Q.C. 26\%, e-v. 0.048, for. Id. 100\% (Figure 1B);

Bungarotoxin (V31) Bungarus multicinctus alpha- (id. Y17057.1) Q.C 31\%, e-v.0.092 for. Id $89.47 \%$ (Figure 1C); 


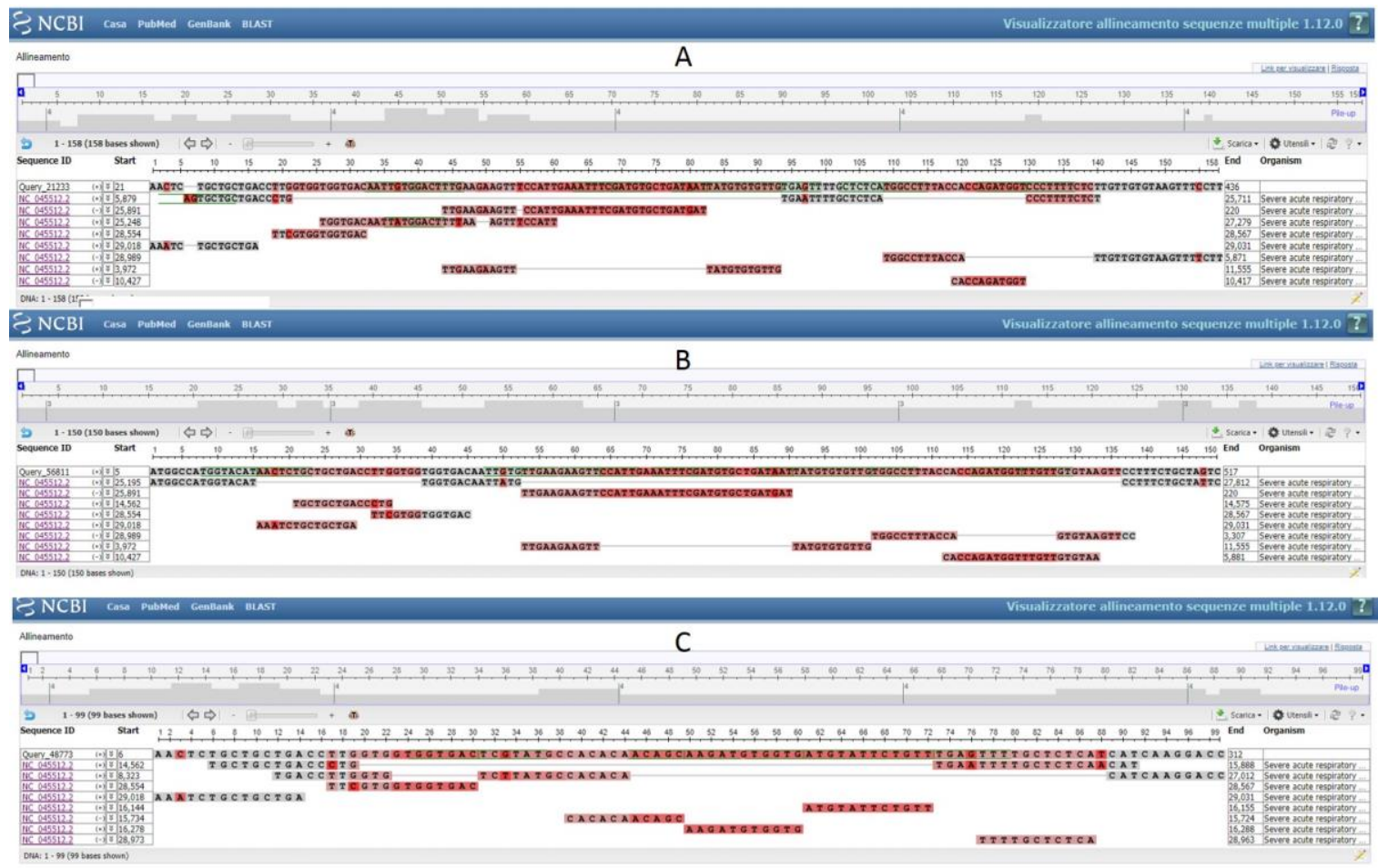

Figure 1. -B. Multicinctus peptide 1 similar to neurotoxin (NL1) (X64593.1), Query cover 32\%, e-value 0.5, 100\% identity percentage (Figure 1A); Cardiotoxin VII Naja atra (id. U42584.1), Q.C. 26\%, e-v. 0.048, for. Id. 100\% (Figure 1B); Bungarotoxin (V31) Bungarus multicinctus alpha- (id. Y17057.1) Q.C 31\%, e-v.0.092 for. Id 89.47\% (Figure 1C).

Clone pGEMT-BMNTL4 Bungarus multicinctus mRNA (id. AJ007764.1) Q.C. 33\%, e-v 0.092 for. Id. $100.00 \%$ (Figure 2.D);

Alpha- bungarus3ftx Type II (id. CAB51841.1) Query 32\%, e-v. 0.14, per.Id. 93.75\% (Figure 2.E);

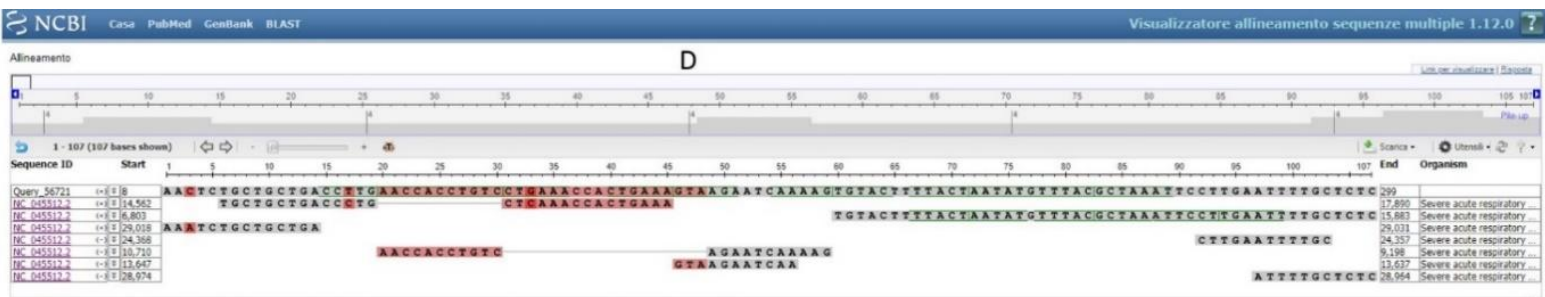

Fig 2.D Clone pGEMT-BMNTL4 Bungarus multicinctus AJ007764.1

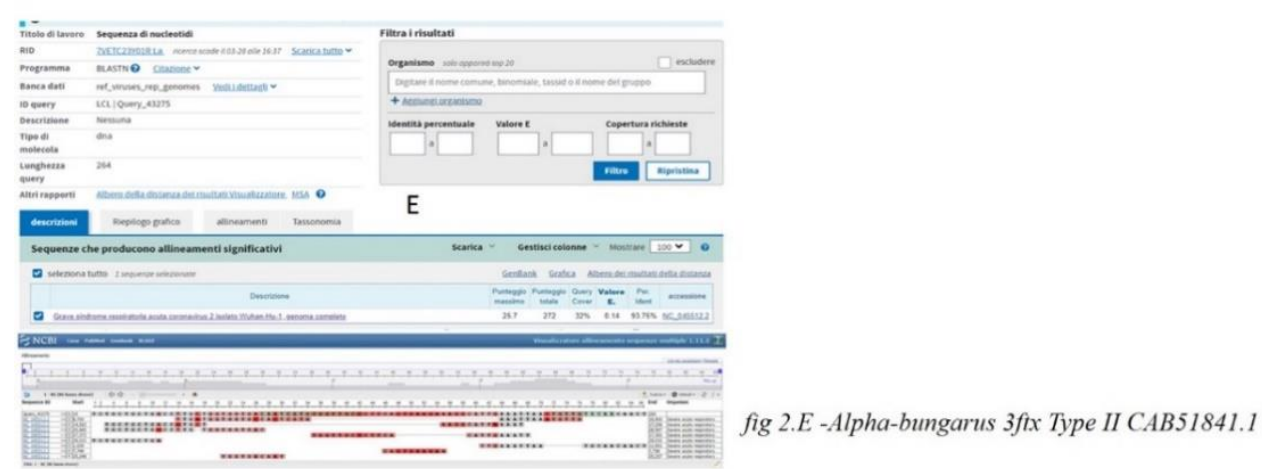

Figure 2. Bungarus multicinctus mRNA (Clone pGEMT-BMNTL4 id. AJ007764.1) Q.C. 33\%, e-v 0.092 for. Id. 100.00\% (Figure 2.D); Alpha- bungarus3ftx Type II (id. CAB51841.1) Query 32\%, e-v. 0.14, per.Id. $93.75 \%$ (Figure 2.E). 
Beta bungaratoxin B2a chain Bungarus ca. (id. AAL30066.1) Q.C. 54\%, e-v. 0.19, per.id. 93.33\% (Figure 3 F); 3.G);

Prothrombin activator Tropidechis ca. (id. DQ533832.1) Q.C. 32\%, e-v. 0.003, per.id. 100\% (Figure

PLA2 gene for phosp. Laticauda la. (id. AB062441.1) Q.C. 16\%, e-v. 0.2, per.id. 89.47\% (Figure 3.H);

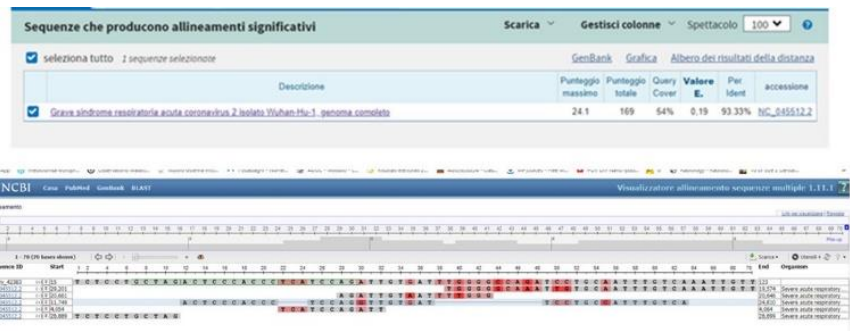

Fig 3. F-Beta bungaratoxin B2a chain Bungarus ca AAL30066.1

Fig. 3 G-Prothrombin activator Tropidechis ca. DQ533832.1 query $32 \%$ ed e-value 0.003

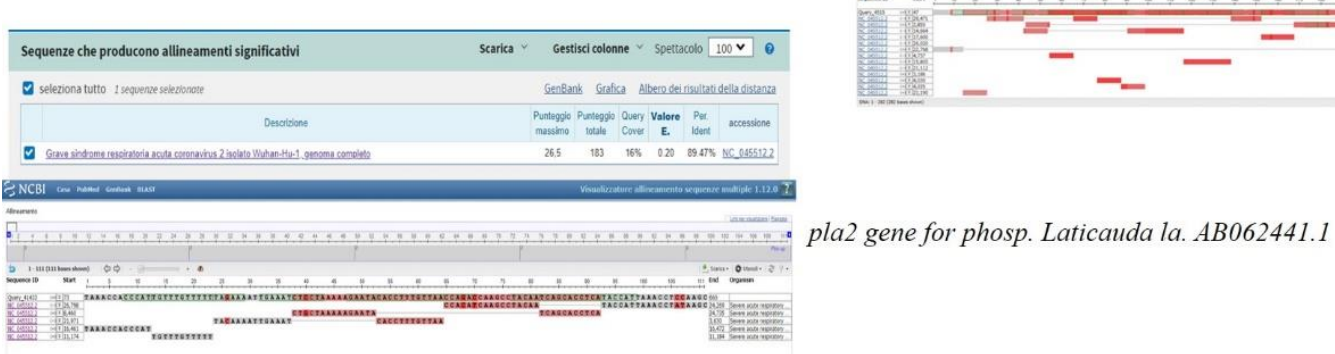

Figure 3. Bungarus ca Beta bungaratoxin B2a chain. (id. AAL30066.1) Q.C. 54\%, e-v. 0.19, per.id. 93.33\% (Figure 3 F); Tropidechis ca. Prothrombin activator (DQ533832.1) Q.C. 32\%, e-v. 0.003, per.id. 100\% (Figure 3.G); Laticauda la PLA2 gene for phosp.. (id. AB062441.1) Q.C. 16\%, e-v. 0.2, per.id. $89.47 \%$ (Figure $3 . \mathrm{H})$.

Ophiophagus precursor gene hannah Ohanin (king naja), (DQ103590.1) Q.C. 16\%, e-v.0.056, id. $100 \%$ (Figure 4.);

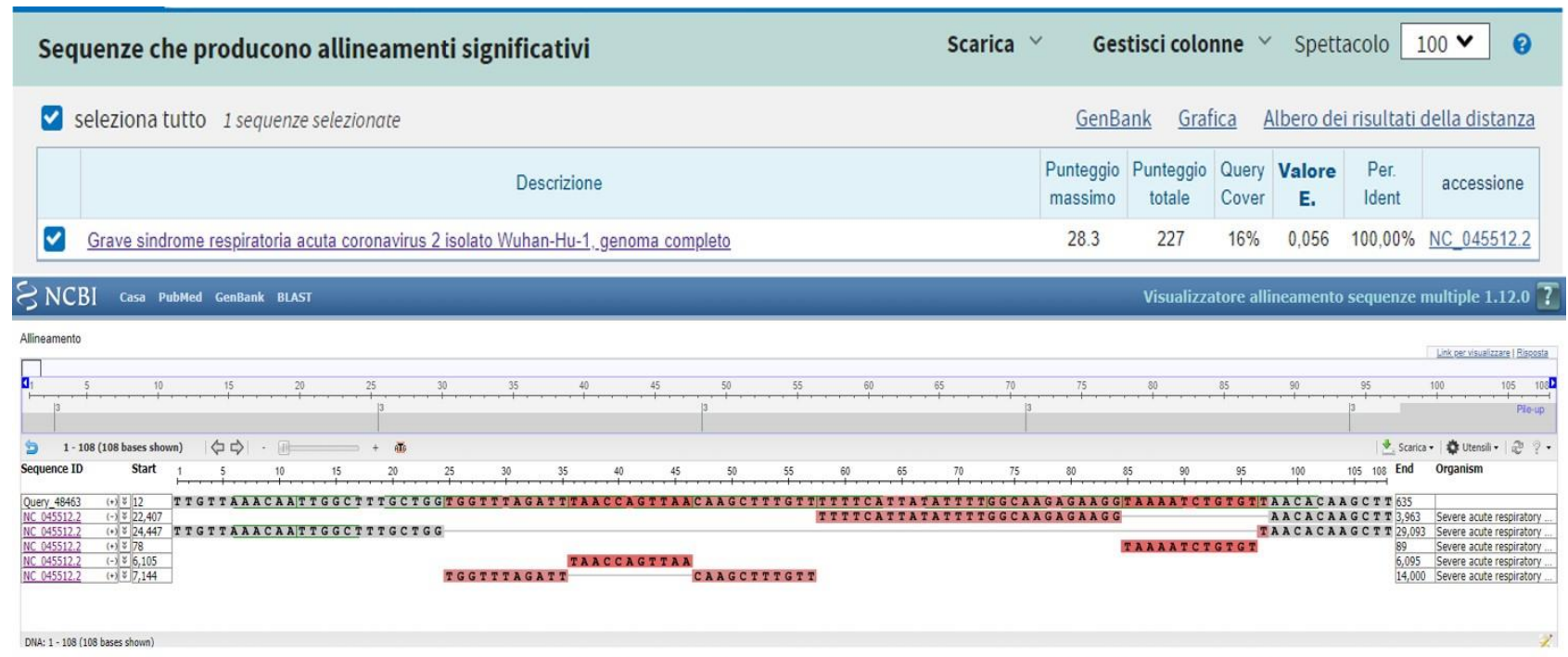

Fig.4 - gene precursor di Ophiophagus hannah Ohanin (king naja), DQ103590.1 
Figure 4. Ophiophagus hannah Ohanin (king naja) Gene precursor of, (DQ103590.1) Q.C. 16\%, ev.0.056, id. $100 \%$

The deductive/inductive process.

Neurotoxins are divided into two categories: short chain (60-62 amino acids, four-disulfide bonds) and long chain (66-74 amino acids or more and with five-disulfide bonds). The long-chain peptide binds with greater affinity to the alpha- $7 \mathrm{nACh}$ receptors, through the fifth disulfide bond (neuronal k-bungarotoxin) (28). The family of three-finger toxins (about $703 F T x$ s: cardiotoxic and neurotoxic) are 60-74 amino acid residues polypeptides composed. They show different functionality while have a preserved structure. A distinct structural feature is the unique fold, consisting of three rings (stranded), which emerge from a hydrophobic globular core. The four or five disulfide bonds, obliged with some Cys, stabilized three-dimensional structure. Short rings length, conformations and amino acid residue variations are responsible of their distinct biological functions (77). Fasciculine, with their loops and key amino-acids placed on the outside, creates a complex fasciculine-Ache (78). Toxic $A 2$ phospholisis interacts with acetyl-choline nicotinic receptors (27.36). Almost all these toxins bind with high affinity to the acetylcholine nicotinic receptors (AChR) periphery (28). In some studies, alpha carbotoxin amino acids, Ala-28, Lys-35 and Cys-26-Cys-30 have been seen to selectively bind to alpha 7-AChR, while Lys-23 and Lys-49 bind exclusively to AChR Torpedo. Therefore, alpha-Cbtx binds to two subtypes of AChR using both common and specific residues. The link with toxins probably occurs in neuronal and muscular AChR homologous regions $(67,68)$. Probably, a small ring cycled, with a solid disulfide bond and uniquely present in long-chain toxins, could serve as discriminating element, and a small toxin fold structural deviation can generate a unique discriminatory recognition for some receptor subtypes (69). The Three-finger toxins (3FTxs) block post-synaptic transmission through nicotinic receptors (nAARTr). The three-fingered toxin fold plasticity has evolved optimally to use different functional groups combinations, to generate a targets specificity panoply, to discern fine differences between nAChR subtypes. (33). Alpha-bungarotoxin binds to skeletal muscle nicotinic receptors, block neuromuscular transmission (29). Protamine and polylisine are even more potent than B. multicinctus cardiotoxin inhibiting cholinesterase (32.34). Some toxins have a basic phospholipase unit and possess anticoagulant properties; others induce the platelets aggregation; others instead inhibit it. Many "curare" toxins with threefingered structure recognize a protein that binds to acetylcholine and block nicotinic receptors in skeletal muscles producing flaccid paralysis. Other toxins recognize acetylcholine receptors located on neurons. Cardiotoxins damage heart function; they depolarize numerous excitable cells membranes and are powerful cytotoxins. In total, toxins with three-finger structure exercise at least seven different functions. Certain snake poisons toxins don't belong to any of the two structural categories mentioned. Sarafotoxins are 21 amino acids vasoconstrictor peptides, with two disulfide bonds. Finally, the disentegrins possess the preserved sequence arginine-glicin-acid aspartic and inhibit the platelets aggregation and recognize proteins glycoprotein IIb/IIIa (70-75). Selectively bind the integrin receptors on platelets surface and other cells. Tirofiban and Eptifibatide are derived and used as antithrombotic agents (77). Protein toxins activate prothrombin, including Xa coagulation factor (FXa) (79). The Kunitz type inhibitor works inhibiting serine and proteases (e.g. plasmin, kallikrein, trypsin). It interferes with the coagulation cascade and fibrinolysis $(76,77)$. In acute coronary syndrome there is a high chemokine CXCL12 and its CXCR4CXCR7 receptor platelet surface expression and it influences prognosis (80).

Most toxins are, however, aChE inhibitors (37). Low aChE concentration is associated with mortality increased in patients with reduced left ventricular ejection fraction for any reason, and the mortality risk increases for all causes related to the aChE functionality (38). While the cholinesterase may be similar by gender and age, not so the activity of erythrocytes cholinesterase may variable (39). The prevalence of $B u C h E$ deficiency is highest among European: the population has a partial deficiency between 3.4 and $4 \%$. The most severe form prevalence is estimated at 1: 100,000. BuChE deficiency has hereditary and nonhereditary causes. The genetic defect, mutations in BCHE-Gen, is inherited in an autosomal recessive gene (locus E1 in the chromosomal region 3q26.1-q26.2). The acylation/catalytic site offers the Ser, His and Glu triad for aChE and Ser, his and Glu for the BuChE, suitable for inhibition. ACh substrates, as well as its inhibitors, can covalently bind to the active site following the attack on the substrate's carboxylic group by the hydroxyl anion Ser. The choline / cationic middle site within the aChE and 
BuChE site contains amino acids (Trp and Tyr in aChE, Trp and Wing in BuChE) that interact with the quaternary amino group within the ACh residue to optimally orient the molecule. The blocking site and, therefore, the substrate approach by the catalytic triad within the acylation/catalytic site will clearly reduce the aChE and/or BuChE ability to metabolize ACh and prolong its drug action $(55.56,57,58,59,60,61,62,63.64)$.

In all disorders associated with a cortical presynaptic cholinergic deficiency, reflected with a choline acetyl-transferase extensive loss (Alzheimer's disease, Parkinson's disease and Down syndrome) there is a substantial nicotine binding nicotinic receptor reduction, in the bond ( $3 \mathrm{~h})$. In contrast, reductions both muscarinic subtypes (M1 and M2) are moderate in Alzheimer's disease while significantly increase (apparently not in relation to anti-cholinergic drug treatment) in Parkinson's disease and in cases with dementia but not in those without. (81).

A non-hereditary deficiency of BuChE can occur in pregnancy, in newborns or in chronic infections, malnutrition, liver diseases and cancers, iron deficiency anemia and drugs such as cocaine, morphine, codeine, succinyl-choline, OP poisoning" or from excess acetylcholinesterase inhibitors (Assumption for PD and AD) cases. Inhibit acetylcholinesterase (aChE) and increase the acetylcholine availability in cholinergic synapses, improves cholinergic transmission in the Alzheimer's disease (AD) pharmacological treatment. On the other hand, the pneumonia cumulative incidence is 51.9 per 1000 cases, or 5.19\%, in subjects over 65 years old, who are in therapy for dementia with cholinesterase inhibitor, galantamine, or rivastigmine (35).

If cholinesterase doesn't increase within 48 hours by intoxication, they are associated with increased mortality due to excess inhibitors (43). They are measured by the dibucaine and fluoride inhibition test. The dibucaine number represents the enzyme activity inhibition value in drug or toxins presence $(41,42)$. The $S 100 B$ serum value are higher in mechanically ventilated patients and serve to predict the need for more aggressive therapy during acute poisoning (42). The two closely homologous enzymes, acetylcholinesterase and butyryl cholinesterase (aChE and BuChE, respectively) are useful as biomarkers in diseases associated with parasympathetic malfunction. Measurements of blood cholinesterase are an easily quantifiable reading for changes in sympathetic/parasympathetic balance, and the implications of changes are understood to be health and disease reading. In our body, two classes of cholinesterase enzymes coexist and possess several cholinergic and non-cholinergic functions, depending on their expression time, position and specific subtype form. Although, aChE also predominates at the neuronal level, BuChE is widely localized and secreted by glial cells. Neurons possess BuChE rather than $\mathrm{aChE}$, and their role in cholinergic function is supported by their placement and/or proximity to choline acetyltransferase (ChAT), the velocity limiter enzyme that catalyzes ACh synthesis. About 10\%-15\% cholinergic neurons inside the human hippocampus and amygdala seem to express BuChE rather than $\mathrm{aChE}$. The $\mathrm{aChE}$ and $\mathrm{BuChE}$ share about $65 \%$ amino acid sequence homology although encoded by different genes on human chromosomes 7 (7q22) and 3 (3q26), respectively. However, both bind and split ACh highly and effectively, with slightly different kinetics, making choline and acetic acid metabolic products identical. The aChE has three residues related to asparagus with carbohydrates and BuChE has nine, which can affect their watery solubility, stability, functional conformation and therefore their biological activity. In addition, both enzymes can form disulfide bond with cysteine defined along their sequence to support their dimerization to generate multiple structural enzymes subunits forms in different brain sites, such as the three globular forms (G forms) that include one (G1), there are mainly two catalytic subunits (G2) or four (G4). The site associated with ACh hydrolysis is internalized in both enzymes. The aChE and BuChE both have an active narrow site, mainly hydrophobic, characterized by X-ray crystallography in the order of $20 \AA$ depth for aChE, in which ACh spreads and then divides (54). ACh is involved in modulation of inflammatory states. Recent data shown that $\mathrm{ACh}$, derived from parasympathetic innervations, inhibits the tumor necrosis factor (TNF-) and interleukin 1 (IL-1) release from macrophages through the nicotinic receptors activation, and the data support an "anticholinergic" inflammatory pathway existence (46-47-48). In the central nervous system, nicotinic and muscarinic receptors subtypes are present on both neurons and glial cells where they mediate motor control, memory regulation, temperature regulation (hyperthermia that is difficult to control), synapse and plasticity functions such as auto- 
heteroreceptors. Within the peripheral nervous system, muscle receptors seem widely involved in several functions, such as smooth muscle contraction, glandular secretion and heart rate regulation (49-50). All cells types within the respiratory tract express muscarinic and nicotinic receptors. Ipratropium and oxitropium (M1/M3 receptors antagonists) and, more recently, tiotropium (nonselective) have an advantage over atropine or other muscarinic antagonists, resulting from their pharmacokinetics; the presence of a quaternary ammonium in their chemical formula allows a local effect that reduces its absorption by bronchus $(51,52,53)$.

\section{Discussion.}

Cholinesterase serves as possible poisoning indicator mediated by the acetyl cholinesterase enzyme inhibition. They are used as liver function functionality index. Their blood values are reduced in organophosphorus intoxication, liver dysfunctions (hepatitis, etc.), cirrhosis, myocardial infarction, acute infections and atypical enzyme phenotypes. Atypical forms are traditionally distinguished with the inhibition dibucaine test resistance or fluoride and so with cholinesterase activity (Woman values: normal: $>75 \%$, heterozygous: 50-75\%, homozygous: $<50 \%$; Man values: normal: $>75 \%$, heterozygous: $50-75 \%$, homozygous: $<50 \%$ ). They are toxins target present in many animal poisons. Those snake toxins (Naja Atra and Bungarus m. and f.) are acetylcholinesterase and particulary Butyryl cholinesterase (pseudo cholinesterase). Some toxins also have similar properties to neuromuscular nicotinic receptor agonists. The toxicity manifests itself in part to an excessive increase in Ach and partly to the nicotinic receptors block: prolonged apnea, cardiocirculatory collapse, heart arrhythmias, ventricular tachycardia, body temperature alteration, electrolyte imbalance (particularly $\mathrm{K}+$ ), butyryl cholinesterase low plasma levels. In the first case the effects are the increase in the action of Acetylcholine consequence (M2 receptors: bradycardic effect with slowing heart activity and reduction of cardiac range, followed by arrhythmic and tachycardic compensation) and with other nonspecific neurosensory symptoms. They result in generalized vasodilation resulting in rapid drop in blood pressure; M3 receptors: huge increase in mucous bronchial secretions with vasoconstriction, increased gastrointestinal motility. Local effects are due to aerosol exposure at the contact point through the eyes or respiratory system or followed by local fluid absorption through the skin or mucous, including gastrointestinal tract. The effects duration is largely determined by the characteristics of the toxin: fat-soluble, whether it should be metabolically activated or not, the toxin-aChE complex stability and the phosphorous enzyme "aging". Eye symptoms include: myosis, eye pain, conjunctive congestion, vision reduction, ciliate spasm and eyebrow pain. As an acute systemic absorption result, myosis is not shown thank to a powerful sympathetic discharge to response hypotension. Other aspects are rhinorrhea and hyperemia; chest constriction sense, breathlessness, bronchus constriction and bronchial secretions increase. Other symptoms will be nausea, vomiting, abdominal cramps and diarrhea. Until we get to the serious effects from high dosage, that are: extreme salivation, involuntary feces and urine emission, sweating, tearing, bradycardia, hypotension, arrhythmias and cardiocirculatory collapse. Nicotinic actions on neuromuscular junctions are muscle fatigue and general weakness, involuntary contractions, spread fasciculations and finally marked weakness increase and respiratory muscles paralysis. The effects on the Snc are confused state, ataxia, verbal confusion, reflexes loss, Cheynestokes breath, convulsions, coma and respiratory paralysis. The effects on medulla oblongata vasomotor and other cardiovascular centers lead to hypotension with fibrillation and reflex tachycardia. The muscarinic and nicotinic effects of the CNS all contribute to respiratory impairment. These included laryngospasm, bronchus-constriction, increased secretions, impaired diaphragm and intercostal muscles movements and respiratory depression. Blood pressure can drop to low levels due to irregularities in heart rhythm, effects caused by hypoxemia and often antagonized by assisted ventilation lung. Nonspecific symptoms can be anosmia and dysgeusia. Olfactory dysfunction is an early Parkinson's disease 'preclinical' sign, often precedes years and remains the only symptom before disease diagnosis that is confirmed when now more than $80 \%$ GABAergic neurons are lost. The dysgeusia and anosmia pathophysiological and neuronal mechanism in Parkinson's disease (cholinergic pathways at higher levels) are still being studied. It should be remembered that these 
neuronal pathways do not pass through the thalamic nucleus but relate directly to the Hippocampus/Amygdala complex.

\section{Considerations.}

Blastn alignment suggests a good correlation between the genetic sequence of Covid-19 and bungaro-toxins, phospholipase A2 and the similar prothrombin activator protein. The snake can be considered as an intermediate natural host (axiom 1,2,3,8,9 respected) and age variables, phenotype, sex, drugs intake, pre-existing pathologies, special work exposures (health workers, pesticide growers, etc.) may be related and dependent to the viral charge concentration, toxins released and both immune system and cholinesterase functionality (axioms 4,5,6,7 respected). It is not so much essential to know the intermediate host as to understand if the virus can express similar properties. The crazy hypothesis, initially defined as "Absurd - reductio ad absurdum", is very likely to be true.

Below is suggested (Figure 6) a new Covid-19 Virus dual pathogenic model, evolved. It is a respiratory virus like a "Trojan-horse", which in passing through the intermediate host has probably acquired, randomly and naturally, simple or small gene sequences (micro-rna?). Such sequences may be similar, but also split (gene recombination model in natural way). They will probably be transcribed like the originals by 3D globular profile, with same folds number of, charges, the sulfide bond number, mostly for the key amino acids position along the protein chain. They don't necessarily must have the same amino acid length. They can be shorter provided they adapt with at least two or more faces to the binding site receptors, respecting the loops rules, electrostatic charges and key amino acids who are necessary at binding receptor interface. These molecules synthesis is defined as "factors". We could imagine this mechanism very similar to the atomic orbitals Aufbau principle, with the electron's distribution according to the Hund rule (84). The interaction factors and receptors give strength to the viremia. We call these molecules as "The Zero Point Factors". It generates a circuit (loop): faster the viremia and replication are, more factors/toxins are translated, and more viremia will increase thanks to the latter action on the immune system. On the other hand, the immune system also has significant interindividual variations in age, genetics, pathologies, etc. Longer time the immune system takes to produce antibodies against virus, more factors are released in the circulation and more the immune system is blocked by the viremia and factors double synergistic action. In this model, the decisive conditions are the exposition time, the initial and/or repeated viral load (number viral particles/ Inhaled air volume). More longer exposition, occasional or not, simple or repeated, more higher the started viral load is and more the outcome will be subordinate to the toxic state achieved, to the immune system efficiency, to the efficacy enzymes (aChE and buChE) and the antiviral or support therapies adopted. The virus uses its greed for ACE2 receptors. Zero-point Factors would act at the level of central and peripheral cholinergic synapses, and on nicotinic receptors, on clotting factors by enhancing the pathogenicity of the COVID-19 virus. Subjects would therefore be at greater risk: any with ACE2r over-expression (taking drugs-inib. Ace.); health worker, for the increase in exposition time; a low immune system (oncology, autoimmune diseases, treatment with immunosuppressants, etc.) subjects; heart and respiratory diseases (for both toxicity factors produced and viremia) patients; lower levels or altered functioning of cholinesterase (different by age, gender, race and phenotype); neuronal diseases such as Parkinson's (PD), Alzheimer's (AD), etc. or autoimmune such as Myasthenia Gravis patients that being treated with acetylcholinesterase inhibitors; growers with seasonal exposition to organophosphorus (pesticides) and. The possibility of a single factor showing dose/dependent receptor affinity first towards the cholinesterase and then, increased doses, dependent viremia, towards the nicotinic receptors with blocking action is not excluded. The outcome would depend on the dual pathogen action mechanism: on the one hand the elements of viremia and on the other the toxicity factors. 
Covid-19- Zero Point Factors TheoryDouble Pathogenic model
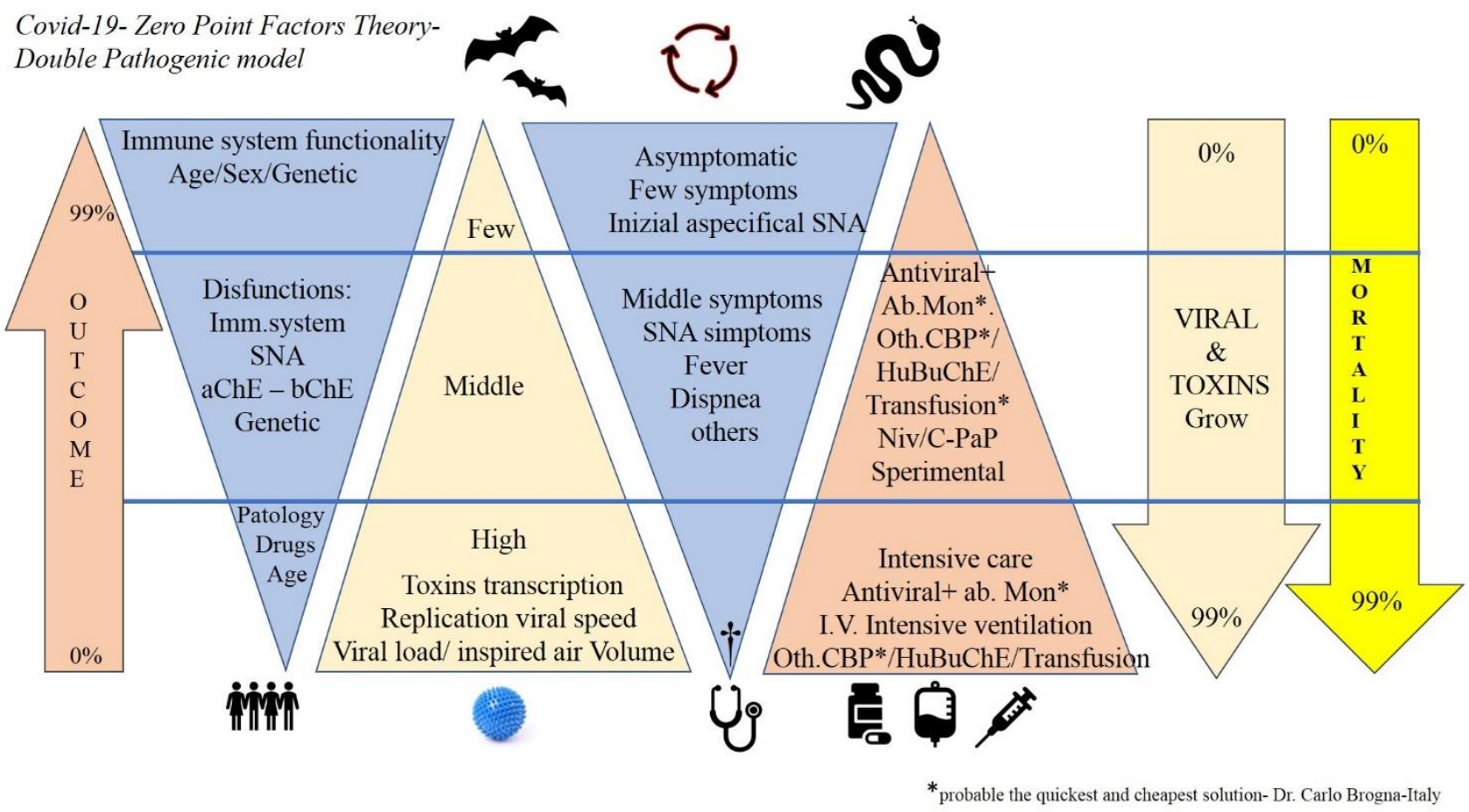

Figure 6. New probable double pathogenic model (M.D.Carlo Brogna).

\section{Conclusions.}

The actual therapies practiced are: Lopinavir/ Ritonavir, Chloroquine, Remdesivir, Ribavirin Synthetic, Oseltamivir, Penciclovir/Acyclovir, Ganciclovir, Favipiravir $(2,30)$. The actual therapies in trial are: Darunavir/cobicistat; Lopinavir/ritonavir; Tocilizumab (83).

Toxins are generally weakly immunogenic and therefore often not effectively targeted by current polyclonal antivenom therapies (31). NAChR imitations, also known as acetylcholine binding proteins (AChBPs), can capture toxins and thus be used to prevent neurotoxic envenoming (31). While waiting for a vaccine solution against the surface virus antigens, valid until the virus will change its recognition characteristics, it would be appropriate to evaluate the suggested data and consider convalescent blood products (CBPS) since they would act not only against the pathogen Covid-19 but also towards the likely factors produced by it (22). The human cholinesterase derivative huBuChE administration ( $\mathrm{im}$ ) (40) could be studied as an adjuvant. The antibodies monoclonal use, probably directed either towards the virus surface antigens and/or towards the foreign factors transcribed from it, could be an immediate solution and allow to contain the mortality rates. Some authors studying the of Pyridostigmine bromide and huperzine A use against nerve agent poisonings (GD), have implemented a fast protocol for cholinesterase dosage, with the full blood test WRAIR WBAIR, US patent No. 6,746,850 help (45). Both the latter and the feature test, dibucaine inhibition (41-42) could be associated with the nasal and/or oropharynx swab (time and sensitive operator), could be used as a criterion and parameter to define the hospitalization and patients outcome, to stratify the risk and to integrate therapeutic possibilities. Such tests could be a valuable tool for interception, monitoring and clinical evaluation. The S100 protein could be used to test outcomes patients during the mechanical ventilation (42). The genesis, "zero point", identification remains a crucial and fundamental moment. The syllogism is simplified in the following scheme: Beta coronaviruses hold " $\mathrm{X}$ " with them; some snakes produce "Y* Z"; covid-19 certainly has an "intermediate host" ; Mr. Blast says that covid-19 and snake toxins are "probably related"; The Covid-19 virus is a beta coronavirus and has " $\mathrm{X}+\mathrm{Y}^{*} \mathrm{Z}$ ". The result is final syllogism: the Covid-19 virus can be a beta coronavirus with toxic factors. It may seem like a basic logic, but the blast data integrated with the observations suggests that conclusion. The autonomic nervous system study is complex and full of molecular, sub-molecular, atomic mechanisms, still unknown. Current knowledge is essential to better differentiate and catalogue the symptoms manifestation. Magnificent ancestral system signs and/or symptoms, while going 
unnoticed, may allow for a reverse deductive and inductive process, that is, from the end to the likely beginning. Clinical trials, research, patterns analysis acquired by the animal and plant kingdom historical knowledge and how they interact with other living beings can help as well as the medical, mathematical, statistical, epidemiological, physical and chemical branches integration in better understanding our autonomous system and its functions.

\section{References:}

1. Arinjay Banerjee, et All in Viruses 2019,11,41;(doi: 10.3390/v11010041) “Bats and Coronaviruses";

2. Yan-Rong Guo1t et All (The origin, transmission and clinical therapies on coronavirus disease 2019 (COVID-19) outbreak - an update on the status- A novel coronavirus from patients with pneumonia in China, 2019. N Engl J Med. 2020;382(8): 727-33);

3. Giovanetti M, Benvenuto D, Angeletti S, Ciccozzi M. The first two cases of 2019-nCoV in Italy: where they come from? J Med Virol. 2020:1-4. Paraskevis D, Kostaki EG, Magiorkinis G, Panayiotakopoulos G, Sourvinos G, Tsiodras S. Full-genome evolutionary analysis of the novel corona virus (2019-nCoV) rejects the hypothesis of emergence as a result of a recent recombination event. Infect Genet Evol. 2020; 79:104212;

4. Zhou P et All (A pneumonia outbreak associated with a new coronavirus of probable bat origin. Nature 2020;

5. Liu Z, Xiao X, Wei X, Li J, Yang J, Tan H, et al. Composition and divergence of coronavirus spike proteins and host ACE2 receptors predict potential intermediate hosts of SARS-CoV-2. J Med Virol. 2020;

6. Wu F, Zhao S, Yu B, Chen YM, Wang W, Song ZG, et al. A new coronavirus associated with human respiratory disease in China. Nature. 2020;

7. de Wit E, van Doremalen N, Falzarano D, Munster VJ. SARS and MERS: recent insights into emerging coronaviruses. Nat Rev Microbiol. 2016;14(8):523-34;

8. Song $\mathrm{Z}, \mathrm{Xu} \mathrm{Y}, \mathrm{Bao} \mathrm{L}$, Zhang $\mathrm{L}, \mathrm{Yu} \mathrm{P}, \mathrm{Qu} \mathrm{Y}$, et al. From SARS to MERS, thrusting coronaviruses into the spotlight. Viruses. 2019;11(1): E59. https:/doi.org/10.3390/v11010059 e Cui J, Li F, Shi ZL. Origin and evolution of pathogenic coronaviruses. Nat Rev Microbiol. 2019;17(3):181-92;

9. Angeletti S, Benvenuto D, Bianchi M, Giovanetti M, Pascarella S, Ciccozzi M. COVID-2019: the role of the nsp2 and nsp3 in its pathogenesis. J Med Virol.2020;

10. Zhang L, Shen FM, Chen F, Lin Z. Origin and evolution of the 2019 novel coronavirus. Clin Infect Dis. 2020. https://doi.org/10.1093/cid/ciaa112 [Epub ahead of print];

11. Jia HP, Look DC, Shi L, Hickey M, Pewe L, Netland J, et al. ACE2 receptor expression and severe acute respiratory syndrome coronavirus infection depend on differentiation of human airway epithelia. J Virol. 2005;79(23): 14614-21;

12. Wan Y, Shang J, Graham R, Baric RS, Li F. Receptor recognition by novel coronavirus from Wuhan: an analysis based on decade-long structural studies of SARS. J Virol. 2020;

13. Tortorici MA, et al. 2019 Veesler D. Structural insights into coronavirus entry. Adv Virus Res. 2019; 105:93116 ;

14. Sawicki SG, Sawicki DL. Coronavirus transcription: a perspective. Curr Top Microbiol Immunol. 2005; 287:31-55;

15. Hussain S, Pan J, Chen Y, Yang Y, Xu J, Peng Y, et al. Identification of novel subgenomic RNAs and noncanonical transcription initiation signals of severe acute respiratory syndrome coronavirus. J Virol. 005;79(9):5288-95. 36;

16. Perrier A, Bonnin A, Desmarets L, Danneels A, Goffard A, Rouille Y, et al. The C-terminal domain of the MERS coronavirus $\mathrm{M}$ protein contains a trans-Golgi network localization signal. J Biol Chem. 2019;294(39):14406-21;

17. Chen C, Zhang XR, Ju ZY, He WF. Advances in the research of cytokine storm mechanism induced by Corona Virus Disease 2019 and the corresponding immunotherapies. Zhonghua Shaoshang Zazhi. 2020;36(0): E005;

18. Liu Y, Zhang C, Huang F, Yang Y, Wang F, Yuan J, et al. 2019-novel coronavirus (2019-nCoV) infections trigger an exaggerated cytokine response aggravating lung injury. 2020;

19. Liu Q, Wang R, Qu G, Wang Y, Liu P, Zhu Y, et al. General anatomy report of novel coronavirus pneumonia death corpse. J Forensic Med. 2020;36(1):19-21.)

20. Kawai T, Akira S. The role of pattern-recognition receptors in innate immunity: update on toll-like receptors. Nat Immunol. 2010;11(5):373-84; 
21. Wei Ji et al. 2020 | Wei Wang | Xiaofang Zhao | Junjie Zai | Xingguang Li (Cross-species transmission of the newly identified coronavirus 2019-nCoV) J Med Virol. 2020; 92:433-440;

22. Convalescent plasma: new evidence for an old therapeutic tool? Giuseppe Marano1, Stefania Vaglio1,2, Simonetta Pupella1, Giuseppina Facco1,3, Liviana Catalano1, Giancarlo M. Liumbruno1, Giuliano Grazzini1 Blood Transfus 2016; 14: 152-7 DOI 10.2450/2015.0131-15;

23. Dawei Wang, Bo Hu 1, Chang Hu , Fangfang Zhu , Xing Liu , Jing Zhang, Binbin Wang, Hui Xiang , Zhenshun Cheng, Yong Xiong, Yan Zhao, Yirong Li, Xinghuan Wang , Zhiyong Peng Affiliations expand PMID: 32031570 PMCID: PMC7042881 (available on 2020-08-07) DOI: 10.1001/jama.2020.1585 JAMA 2020 Feb 7[Online ahead of print Clinical Characteristics of 138 Hospitalized Patients With 2019 Novel Coronavirus-Infected Pneumonia in Wuhan, China;

24. Huang, Yeming Wang, Xingwang Li, Lili Ren, Jianping Zhao, Yi Hu, Li Zhang, Guohui Fan, Jiuyang Xu, Xiaoying Gu, Zhenshun Cheng, Ting Yu, Jiaan Xia, Yuan Wei, Wenjuan Wu, Xuelei Xie, Wen Yin, Hui Li, Min Liu, Yan Xiao, Hong Gao, Li Guo, Jungang Xie, Guangfa Wang, Rongmeng Jiang, Zhancheng Gao, Qi Jin, Jianwei Wangt, Bin Caot Clinical features of patients infected with 2019 novel coronavirus in Wuhan, China Chaolin Lancet 2020; 395: 497-50;

25. 'Yan-Rong Guot, Qing-Dong Caot, Zhong-Si Hongt, Yuan-Yang Tan, Shou-Deng Chen, Hong-Jun Jin, KaiSen Tan, De-Yun Wang and Yan Yan) Mil Med Res. 2020; 7: 11 The origin, transmission and clinical therapies on coronavirus disease 2019 (COVID-19) outbreak - an update on the status;

26. Burnouf T, Seghatchian J Transfus Apher Sci. Ottobre 2014; 51 (2): 120-5. Ebola Virus Convalescent Blood Products: Where We Are Now and Where We May Need to Go;

27. Vulfius et all (, PLOS ONE I DOI: 10.1371/journal.pone.0115428 December 18, 2014;

28. Denis Servent $\$$, Valérie Winckler-Dietrich $\ddagger$, Hai-Yan Hu, Pascal Kesslerł, Pascal Drevetł, Daniel Bertrand and André Ménezł Only Snake Curaremimetic Toxins with a Fifth Disulfide Bond Have High Affinity for the Neuronal $\alpha 7$ Nicotinic Receptor* (J. Biol. Chem. 272, 24279-24286;

29. Chiappinelli r. e all. (Dipartimento di Farmacologia, Harvard Medical School, Boston, Massachusetts 02115 Comunicato da Stephen W. Kuffler, il 21 marzo 1978;

30. Liying Dong, Shasha Hu, Jianjun Gao Discovering drugs to treat coronavirus disease 2019 (COVID-19) Drug Discoveries \& Therapeutics. 2020; 14(1):58-60;

31. Laura-Oana Albulescu et all Front Pharmacol. 30 lug 2019; 10: 848. doi: 10.3389 -A Decoy-Receptor Approach Using Nicotinic Acetylcholine Receptor Mimics Reveals Their Potential as Novel Therapeutics Against Neurotoxic Snakebite;

32. Di Shoei-Yn shiau lin, chin liao e c. Y. Lee (Biochimica. J. (1977) 161.229-232 Mechanism of cardiotoxin, Protaxin and Polylisin anticholinesterase activities);

33. Selvanayagam Nirthanan e Matthew C.E. Gwee1 (Tre dita -Neurotossine e il recettore dell'acetilcolina nicotinica, Quarant'anni dopo Pharmacol Sci 94, 1 - 17 (2004);

34. Da c. y. lee, c. c. Chang e k. kamijo 1955 (Il Farmacologico Institute, College of Medicine, National Taiwan Univer8ity, Taipei, Formo8a, Cina, e il Dipartimento di Fisiologia e Farmacologia, Graduate School of Medicine, Univeraity di Pennsylvania, Philadelphia, Pennsylvania, Stati Uniti -Ricevuto il 23 luglio 1955) Cholinesterase Inattivazione di Snake Venoms;

35. Edward Chia-Cheng Lai, et all (Comparative risk of pneumonia among new users of Cholinesterase Dementia Inhibitors J Am Geriatr Soc. 2015 maggio; 63(5): 869-876. doi: 10.1111/jgs.13380);

36. Catherine A. et all (Inhibition of nicotinic acetylcholine receptors, a new facet in the Pleiotropic Activity of Snake Venom Phospholipases A2PLOS ONE I DOI: 10.1371/ journal. pone.0115428 December 18, 2014);

37. Vivitri Prasasty, Muhammad Radifar ed Enade Istyastono (Natural peptides in targeting for the discovery of Acetilcholinesterase drugs- Molecules 2018, 23, 2344; doi: 10.3390/molecules23092344www.mdpi.com/journal/molecules);

38. Masahiro Seo et all, (Prognostic Significance of Serum Cholinesterase Level in Patients With Reduced, MidRange and Preserved Left Ventricular Ejection Fraction With Acute Decompensated Heart Failure: A Prospective Study in Osaka Prefectural Acute Heart Failure Registry (OPAR) Circulation. 2018;138: A12826;

39. AA.VV (Influence of age, sex and oral contraceptives on the activity of cholineesterase in human blood Clinical Chemistry, Volume 21, Issue 10, 1 settembre 1975, Pagine 1393-1395);

40. Mumford H (Human Plasma-Derived BuChE as a Stoichiometric Bioscavenger for Treatment of Nerve Agent Poisoning Chem Biol Interact , 203 (1), 160-6 2013 Mar 25; 
41. P. DE LONLAY (Ottobre 2006 ORPHA:132) ultima revisione: Butyrylcholinesterase deficiency Orphanetwww.orpha.net> consor> cgi-bin.;

42. T. Yardan, et all (The Role of Serum Cholinesterase Activity and S100B Protein in the Evaluation of Organophosphate Poisoning) Hum Exp Toxicol, 32 (10), 1081-8 Ottobre 2013;

43. Chen et all 2008 (Prognostic value of sieric cholineesterase silky activities in patients poisoned by organophosfati j. ajem.2008.07.006);

44. Shenhar et all. (Cholineesterase as biomarkers for parasympathetic dysfunction and inflammation-related diseaseJ Mol Neurosci, 53 (3), 298-305 Lug 2014);

45. Gordon Richard K Gordon, Julian R Haigh, Gregory E Garcia, Shawn R Feaster, Michael A Riel, David E Lenz, Paul S Aisen, Bhupendra P Doctor Affiliations expand et all (Oral administration of pyridostigmine bromide and Huperzine A protects whole human blood cholineesterases from ex Vivo exposure to Soman Chem Biol Interact, 157-158, 239-46 15 dicembre 2005);

46. Nigel H. Greig, Marcella Reale, e Ada Maria Tata (New advances in pharmacological approaches to the cholinergic system: a overview of the mucarinic receptor ligands and the Cholinesterase Recente Pat CNS Drug Discov. 2013 ago; 8 (2): 123-141);

47. (Wessler I, Kilbinger H, Bittinger F, Unger R, Kirkpatrick CJ. The biological role of non-neuronal acetylcholine in plants and humans. Giapponese J Pharmacol. 2001; 85: 2-10.;

48. (Rosas-Ballina M, Tracey KJ. Immune system neurology: Neural reflexes regulate immunity. Neurone. 2009; 64: 28-32.);

49. Ragheb F, Molina-Holgado E, Cui QL, et al. Caratterizzazione farmacologica e funzionale dei sottotipi di recettori muscarinici nello sviluppo di oligodendrociti. J Neurochem. 2001; 77: 1396-1406;

50. De Angelis F, Bernardo A, Magnaghi V, Minghetti L, Tata AM. Sottotipi di recettori muscarinici come potenziali bersagli per modulare la sopravvivenza, la proliferazione e la differenziazione dei progenitori degli oligodendrociti. Dev. Neurobiol. 2012; 72: 713-728;

51. Oki T, Maruyama S, Takagi Y, Yamamura HI, Yamada S. Characterization of muscarinic receptor binding and inhibition of salivation after oral administration of tolterodine in mice. Europ J Pharmacol. 2006; 529:157-163;

52. Racke K, Jurgens UR, Matthiesen S. Control by cholinergic mechanisms. Eur J Pharmacol.2006;533:57-68.; Racke K, Matthiesen S. The airway cholinergic system: physiology and pharmacology. Pulm Pharmacol Ther. 2004; 17:181-198;

53. Keam SJ, Keating GM. Tiotropium bromide. A review of its use as maintenance therapy in patients with COPD. Treat Resp Med. 2004; 3:247-268;

54. Darvesh S, Hopkins D, Geula C. Neurobiology of butyrylcholinesterase. Nat Rev Neurosci. 2003; 4:131138. Darvesh S, Cash MK, Reid GA, Martin E, Mitnitski A, Geula C. Butirylcholinestarase in associated with $\beta$-amyloid plaques in the transgenic APPSW/PSEN1dE9 mouse model of Amlzheimer disease. J Neuropathol Exp Neurol. 2012 Jan; 71:2-14. Darvesh S, Grantham DL, Hopkins DA. Distribution of butyrylcholinesterase in human amygdala and hippocampal formation. J Comp Neurol. 1998; 393:374-390 Darvesh S, Reid GA, Martin E. Biochemical and histochemical comparison of cholinesterases in normal and Alzheimer brain tissues. Curr Alzheimer Res. 2010; 7:386-400;

55. Soreq H, Zakut H. Human Cholinesterases and Anticholinesterases. New York: Academic Press; 1993.; Soreq H, Seidman S. Acetylcholinesterase - New roles for an old actor. Nat Rev Neurosci. 2001; 2:294-302.;

56. Perry EK, Perry RH, Blessed G, Tomlinson BE. Changes in brain cholinesterase in senile dementia of Alzheimer's type. Neuropathol Applied Neurobiol. 1978; 4:273-277;

57. Wright CI, Geula C, Mesulam MM. Neurological cholinesterases in the normal brain and in Alzheimer's disease: relationship to plaques, tangles, and patterns of selective vulnerability. Ann Neurol. 1993; 34:373384.;

58. Eng LF, Uyeda CT, Chao LP, Wolfgram F. Antibody to bovine choline acetyltransferase and immunofluorescent localisation of the enzyme in neurons. Nature. 1974; 250:243-245;

59. Greig NH, Sambamurti K, Yu QS, Perry T, Holloway HW, Haberman F, et al. In: Butyrylcholinesterase its Function and Inhibitors. Giacobini E, editor. London: Martin Dunitz; 2003.pp. 69-90;

60. Greig N, Utsuki T, Yu Q, Zhu X, Holloway HW, Perry T, et al. A new therapeutic target in AD treatment: Attention to butyrylcholinesterase: Cur Med. Res Opinions. 2001; 17:1-7;

61. Greig NH, Lahiri DK, Sambamurti K. Butyrylcholinesterase: an important new target in Alzheimer's disease therapy. International Psychogeriatrics. 2002; 14:77-91; 
62. Masson P, Carletti E, Nachon F. Structure, activities and biomedical applications of human butyrylcholinesterase. Protein Pept Lett. 2009; 16:1215-1224 151;

63. Silman I, Sussman JL. Acetylcholinesterase: how is structure related to function? Chem Biol Interact. 2008; 175:3-10;

64. Sussman JL, Harel M, Frolow F, Oefner C, Goldman A, Toker L, Silman I. Atomic structure of acetylcholinesterase from Torpedo californica: a prototypic acetylcholine-binding protein. Science. 1991; 253:872-879);

65. Antil-Delbeke1, C Gaillard, T Tamiya, P J Corringer, J P Changeux, D Servent, A Ménez Affiliations expand PMID: 10852927 DOI: 10.1074/jbc.M909746199 J Biol Chem 275, 29594-601 2000 Sep 22 Molecular Determinants by Which a Long Chain Toxin From Snake Venom Interacts With the Neuronal Alpha 7nicotinic Acetylcholine Recepto S;

66. Antil, S., Servent, D. e Ménez, A. (1999) J. Biol. Chem. 274, 34851-34858);

67. Ackermann, EJ, Ang, ETH, Kanter, JR, Tsigelny, I. e Taylor, P. (1998) J. Biol Chem. 273, 10958-10964);

68. Osaka, H., Malany, S., Molles, BE, Sine, SM, e Taylor, P. (2000) J. Biol. Chem. 275, 5478-5484;

69. D Servente, G Mourier, S Antil, A Ménez SMED Expansion Affiliates: 10022254 DOI: 10.1016 / s0378-4274 (98) 00307-5 Toxicol Lett, 102-103, 199-203 28 December 1998 How tome toxins curemimetic snakes discriminate against nicotinic acetylcholine receptor subtypes;

70. André Ménez (dal libro La struttura delle tossine degli animali velenosi) LE SCIENZE n. 305, gennaio 1994;

71. STOCKER K. (a cura), Serpents, venins, envenimations, Société Herpétologique de France, Edition Fondation Marcel Mérieux, 1987.;

72. Medical Use of Snake Venom Proteins, CRC Press, Boca Raton, 1990;

73. Harvey, Pergamon Press, 1991Snake Toxins, International Encyclopedia of Pharmacology and Therapeutics, a cura di A. H.;

74. MENEZ A. e altri, in «Proceedings of the Royal Society of Edinburgh», 99B, pp. 83-102, 1992.;

75. BONTEMS F. e altri, in «Science», n. 254, pp. 1521-1523.;

76. R Manjunatha Kini nel "Molecular moulds with multiple missions: functional sites in three-finger toxins Clinical and Experimental Pharmacology and Physiology (2002) 29, 815-822;

77. Aisha Munawar, Syed Abid Ali, Ahmed Akrem and Christian Betzel presentano una review dal titolo "Snake Venom Peptides: Tools of Biodiscovery" Toxins 2018, 10, 474; doi:10.3390/toxins10110474;

78. Harald k.l.et all. Van den born,',3 zoran radic, ${ }^{\prime * \prime}$ pascale marchot,'v4 palmer taylor,' and igor tsigelny's2 "Theoretical analysis of the structure of the peptide fasciculin and its docking to acetylcholinesterase". Protein Science (1995), 4:703-715. Cambridge University Press. Printed in the USA.;

79. Venaa RAO*, Jeremiah S. JOSEPH† and R. Manjunatha KINI*łBiochem. J. (2003) 369, 635-642 (Printed in Great Britain) 635 Group D prothrombin activators from snake venom are structuralhomologues of mammalian blood coagulation factor Xa Veena S1;

80. M. Chatterjee ; M. Manke ; M. Cebo ; J. Rheinlaender ; A. Witte ; T. Schäffer ; M. Lämmerhofer ; O. Borst ; M. Gawaz Recettore piastrinico della chemochina CXCR7 Media un effetto antitrombotico e antitrombinfiammatorio Res Pract Thromb Haemost . Luglio 2018; 2 (Suppl 1): 1-368.Pubblicato online il 5 luglio 2018: 10.1002 / rth2.12125;

81. E.K. Perry, R.H. Perry, C.J. Smith, D. Purohit, J. Bonham, D.J. Dick, J.M. Candy, Cholinergic Receptors inCognitive Disorders J. A. Edwardson and A. Fairbairn Can. J. Neurol. Sci. 1986; 13:521;

82. Manual Goodman \& Gilman. Le basi farmacologiche della terapia di Laurence L. Brunton, Randa HilalDandan, e al. | 2018;

83. Note AIFA Italia dal 11 Marzo in poi https://www.aifa.gov.it/documents/20142/1131319/covid19 sperimentazioni in corso 27.03.2020.pdf/b2391bac-7920-0945-51a1-66db453053cf.

84. Iupac. A compendium of chemical terminology, 2nd ed. (the "Golden Book”). Compiled by AD McNaught and A. Wilkinson. Blackwell Scientific Publications, Oxford (1997). Online version (2019-) created by SJ Chalk. ISBN 0-9678550-9-8. https://doi.org/10.1351/goldbook 